\title{
Apsidal motion in eccentric eclipsing binaries: V871 Aql, V345 Lac, V401 Lac and CR Sct ${ }^{\star}$
}

\author{
M. Wolf ${ }^{1}$, P. Harmanec ${ }^{1,2}$, L. Šarounová ${ }^{2}$, M. Zejda ${ }^{3}$, H. Božić ${ }^{4}$, \\ K. Hornoch ${ }^{1}$, V. S. Kozyreva ${ }^{5}$, T. Hynek ${ }^{6}$, and L. Král ${ }^{6}$ \\ 1 Astronomical Institute, Charles University of Prague, 18000 Praha 8, V Holešovičkách 2, Czech Republic \\ 2 Astronomical Institute, Academy of Sciences, 25165 Ondřejov, Czech Republic \\ 3 Nicholas Copernicus Observatory and Planetarium, 61600 Brno, Czech Republic \\ ${ }^{4}$ Hvar Observatory, Faculty of Geodesy, Zagreb University, 10000 Zagreb, Croatia \\ 5 Sternberg Astronomical Institute, State University Moscow, University Avenue 13, Moscow 119899, Russia \\ 6 Johann Palisa Observatory and Planetarium, Technical University Ostrava, 70833 Ostrava-Poruba, Czech Republic
}

Received 22 December 2003 / Accepted 20 February 2004

\begin{abstract}
About thirty new times of minimum light recorded with photoelectric or CCD photometers were obtained for several little studied eccentric-orbit eclipsing binaries V871 Aql $(P=3.0, e=0.13)$, V345 Lac $(7.5,0.46)$, V401 Lac $(2.0,0.16)$ and CR Sct $(4.2,0.09)$. Their $\mathrm{O}-\mathrm{C}$ diagrams were analyzed using all reliable timings found in the literature and in all cases an apsidal motion was either discovered or confirmed. The new values for the elements of the apsidal motion were derived. We find periods of apsidal motion of about 255, 7000, 79.4 and 4400 years for V871 Aql, V345 Lac, V401 Lac and CR Sct, respectively.
\end{abstract}

Key words. stars: binaries: eclipsing - stars: general - stars: fundamental parameters

\section{Introduction}

The study of apsidal motion in detached eclipsing binary systems with eccentric orbits (EEB) is a rewarding area of research, which requires only moderate or small telescopes equipped with a photoelectric photometer or a CCD camera. A detailed analysis of the period variations can be performed using times of minimum light observed throughout the apsidal motion cycle, and from this both the orbital eccentricity and the period of rotation of the periastron can be obtained with a high accuracy. Moreover, this provides independent information for the analysis of the light curves (Giménez 1994). Similar photometric studies of apsidal motion in EEB's were published regularly during the seventies by Helmut Busch, Hartha observatory, and later e.g. by Kh. F. Khaliullin, Moscow University, or J. V. Clausen, Copenhagen University. A catalogue of eclipsing binaries that are suitable for photometric monitoring was provided by Hegedüs (2000) while a catalogue of known binaries with apsidal motion was published by Petrova \& Orlov (1999).

In this paper, we report new results of our observational project initiated more than ten years ago with the main purpose of monitoring the eclipsing binaries with eccentric orbits. In particular, the four northern-hemisphere summer

\footnotetext{
Send offprint requests to: M. Wolf, e-mail: wolf@cesnet.cz

* Partly based on photoelectric observations secured at the Hvar Observatory, Zagreb University, Croatia.
}

objects V871 Aql, V345 Lac, V401 Lac and CR Sct analyzed here are relatively little studied and rather faint eclipsing binaries, although the O-C diagrams for V345 Lac and CR Sct were published by Kreiner et al. (2001). With the exception of V401 Lac, the apsidal motion is announced here for the first time. To the best of our knowledge, no spectroscopic observations have been published for these systems.

\section{Observations of minimum light}

In order to enlarge the number of times of minimum light, new observations for all systems were carried out. New photoelectric photometry was obtained at five different observatories with the aim to secure several new, well-covered primary and secondary minima for all variables:

- Ondřejov Observatory, Czech Republic: the 0.65-m reflecting telescope with the CCD camera SBIG ST-8 or Apogee AP-7 and $R$ or $V$ filters;

- Hvar Observatory, Croatia: the 0.65-m reflecting telescope with the classical photoelectric photometer and Johnsons $U B V$ filters;

- Tien-Shan High Altitude Station of the Sternberg Astronomical Institute, Kazakhstan: 0.70-m telescope with the photoelectric photometer and WBVR filters; 
Table 1. New times of minimum light.

\begin{tabular}{|c|c|c|c|c|c|}
\hline System & $\begin{array}{l}\text { JD Hel.- } \\
2400000 \\
\end{array}$ & $\begin{array}{l}\text { Error } \\
{[\text { day] }}\end{array}$ & Epoch & $\begin{array}{l}\text { Method } \\
\text { filter }\end{array}$ & $\begin{array}{l}\text { Reference } \\
\text { observatory }\end{array}$ \\
\hline \multirow[t]{5}{*}{ V871 Aql } & 49925.300 & 0.001 & 3823.0 & $\mathrm{CCD}, R$ & Ondřejov, this paper \\
\hline & 50242.5359 & 0.0005 & 3930.5 & $\mathrm{CCD}, R$ & Ondřejov, this paper \\
\hline & 51680.4929 & 0.0005 & 4417.5 & $\mathrm{CCD}, R$ & Ondřejov, this paper \\
\hline & 52467.5362 & 0.0001 & 4684.0 & $\mathrm{CCD}, R$ & Ondřejov, this paper \\
\hline & 52492.4813 & 0.0001 & 4692.5 & $\mathrm{CCD}, R$ & Ondřejov, this paper \\
\hline \multirow[t]{9}{*}{ V345 Lac } & 50201.495 & 0.003 & 2517.0 & $\mathrm{CCD}, R$ & Ondřejov, this paper \\
\hline & 50286.53 & 0.02 & 2528.5 & $\mathrm{CCD}, R$ & Diethelm (1996) \\
\hline & 50673.4854 & 0.0002 & 2580.0 & $\mathrm{CCD}, R$ & Ondřejov, this paper \\
\hline & 50713.5572 & 0.0005 & 2585.5 & $\mathrm{CCD}, R$ & Ondřejov, this paper \\
\hline & 51717.47874 & 0.0001 & 2719.5 & $\mathrm{CCD}, V$ & Ondřejov, this paper \\
\hline & 51777.4102 & 0.0004 & 2727.5 & $\mathrm{CCD}, R$ & Ondřejov, this paper \\
\hline & 52239.29095 & 0.0001 & 2789.0 & $\mathrm{CCD}, V$ & Ondřejov, this paper \\
\hline & 52651.3427 & 0.0003 & 2844.0 & $\mathrm{CCD}, R$ & Lelekovice, this paper \\
\hline & 52898.5748 & 0.0001 & 2877.0 & $\mathrm{CCD}, R$ & Ondřejov, this paper \\
\hline \multirow[t]{11}{*}{ V401 Lac } & 48170.268 & 0.001 & -170.0 & pe, $H_{p}$ & Hipparcos, this paper \\
\hline & 48501.7900 & - & 0.0 & pe, $H_{p}$ & Hipparcos, ESA (1997) \\
\hline & 48514.66 & 0.01 & 6.5 & pe, $H_{p}$ & Hipparcos, this paper \\
\hline & 48781.82 & 0.01 & 143.5 & pe, $H_{p}$ & Hipparcos, this paper \\
\hline & 51842.3465 & 0.0002 & 1713.0 & $\mathrm{CCD},-$ & Hegedüs et al. (2003) \\
\hline & 51843.418 & - & 1713.5 & computed & this paper, see text \\
\hline & 52148.5116 & 0.0001 & 1870.0 & $\mathrm{CCD},-$ & Ostrava, this paper \\
\hline & 52464.4306 & 0.0001 & 2032.0 & $\mathrm{CCD}, R$ & Ostrava, this paper \\
\hline & 52891.5075 & 0.0003 & 2251.0 & pe, $U B V$ & Ak \& Filiz (2003) \\
\hline & 52898.4022 & 0.0007 & 2254.5 & pe, $U B V$ & Ak \& Filiz (2003 \\
\hline & 52940.2601 & 0.0003 & 2276.0 & pe, $U B V$ & Hvar, this paper \\
\hline \multirow[t]{10}{*}{ CR Sct } & 45172.388 & 0.005 & 4079.5 & pe, $V$ & Tien-Shan, this paper \\
\hline & 45564.3076 & 0.0005 & 4173.0 & pe, $V$ & Tien-Shan, this paper \\
\hline & 50232.558 & 0.001 & 5286.5 & $\mathrm{CCD}, R$ & Ondřejov, this paper \\
\hline & 50318.4346 & 0.0002 & 5307.0 & $\mathrm{CCD}, R$ & Ondřejov, this paper \\
\hline & 51364.4907 & 0.0002 & 5556.5 & $\mathrm{CCD}, R$ & Ondřejov, this paper \\
\hline & 51777.3707 & 0.0002 & 5655.0 & $\mathrm{CCD}, R$ & Ondřejov, this paper \\
\hline & 51798.33117 & 0.0001 & 5660.0 & $\mathrm{CCD}, R$ & Ondřejov, this paper \\
\hline & 52454.49927 & 0.0001 & 5816.5 & $\mathrm{CCD}, R$ & Ondřejov, this paper \\
\hline & 52802.4640 & 0.0004 & 5899.5 & $\mathrm{CCD}, R$ & Ondřejov, this paper \\
\hline & 52846.41902 & 0.0001 & 5910.0 & $\mathrm{CCD}, R$ & Ondřejov, this paper \\
\hline
\end{tabular}

- Private observatory of K.H. at Lelekovice, Czech Republic: the $0.35-\mathrm{m}$ Newtonian telescope with the CCD camera SBIG ST-6V and $R$ filter;

- Johann Palisa Observatory and Planetarium of Technical University, Ostrava, Czech Republic: the $0.15-\mathrm{m}$ or 0.09-m telescopes with CCD camera Meade Pictor XTE 416 or SBIG ST-7.

The CCD measurements in Ondřejov, Lelekovice and Ostrava observatories were flat-fielded via sky exposures taken at dusk or dawn. Several comparison stars were chosen on the same frame as the variables. No variations in the brightness of these stars exceeding the expected error of measurements were detected (typically $\sigma \simeq 0.005 \mathrm{mag}$ in Ondřejov). No correction for differential extinction was applied, due to the proximity of the comparison stars to the variable and the resulting negligible differences in air mass.

The photoelectric measurements of CR Sct at TienShan observatory were done using the standard photoelectric photometer and $V$ filter of the $W B V R$-photometric system. Concerning this equipment see Khaliullin et al. (1985) for more details. The new times of primary and secondary minima and their errors were determined by fitting polynomials to the data by the least squares and the bisecting chord method and are given in Table 1. Experience shows that this method gives smaller errors than the often used Kwee \& Van Woerden (1956) method. (For instance, the latter method gives HJD $2452239.2898 \pm 0.0056$ for a well-covered 
Table 2. Apsidal motion elements for V871 Aql, V345 Lac, V401 Lac and CR Sct.

\begin{tabular}{cccccc}
\hline \hline Parameter & Unit & V871 Aql & V345 Lac & V401 Lac & CR Sct \\
\hline$T_{0}$ & HJD & $2438637.1489(9)$ & $2431343.5977(7)$ & $2448501.8816(12)$ & $2428069.6810(7)$ \\
$P_{\mathrm{s}}$ & days & $2.9526698(12)$ & $7.4919207(8)$ & $1.9500920(12)$ & $4.1923467(11)$ \\
$P_{\mathrm{a}}$ & days & $2.9527633(12)$ & $7.4919426(8)$ & $1.9502231(12)$ & $4.1923577(11)$ \\
$e$ & & $0.1295(12)$ & $0.456(8)$ & $0.161(7)$ & $0.089(4)$ \\
$\dot{\omega}$ & deg cycle ${ }^{-1}$ & $0.0114(12)$ & $0.00106(10)$ & $0.0242(25)$ & $0.00094(9)$ \\
$\dot{\omega}$ & deg yr yr $^{-1}$ & $1.41(14)$ & $0.052(5)$ & $4.53(45)$ & $0.0082(8)$ \\
$\omega_{0}$ & deg & $178.2(1.2)$ & $238.4(1.0)$ & $16.4(1.1)$ & $68.9(1.3)$ \\
$U$ & years & $255(26)$ & $7000(700)$ & $79.4(8)$ & $4400(450)$ \\
\hline
\end{tabular}

minimum of V345 Lac. Note that the value agrees with the value adopted here within the quoted errors - see Table 1). Only the bottom parts of the eclipses were used. All epochs in Table 1 are calculated from the light elements given in Table 2, the other columns are self-explanatory.

\section{Apsidal motion analysis}

The apsidal motion in all systems was studied by means of an $\mathrm{O}-\mathrm{C}$ diagram analysis. For a more accurate calculation of the apsidal motion rate, the method described by Giménez \& García-Pelayo (1983) and revised by Giménez \& Bastero (1995), was used. This is a weighted least squares iterative procedure, including terms in the eccentricity up to the fifth order. Due to the relatively large values of the orbital eccentricity of some studied systems, we used all terms in our calculation. The relation for the prediction of the times of minimum, used for the minimization by the least-squares method, is also given in Wolf \& Šarounová (1995). There are five independent variables $\left(T_{0}, P_{\mathrm{s}}, e, \dot{\omega}, \omega_{0}\right)$ to be determined in this procedure. The relation between the sidereal and the anomalistic period, $P_{\mathrm{s}}$ and $P_{\mathrm{a}}$, is given by

$P_{\mathrm{s}}=P_{\mathrm{a}}\left(1-\dot{\omega} / 360^{\circ}\right)$

and the period of apsidal motion by

$U=360^{\circ} P_{\mathrm{a}} / \dot{\omega}$.

We have collected all reliable times of minimum light available in the literature. All new precise photoelectric and CCD times of minimum were used with a weight of 10 or 20 in our computation. The current photographic as well as some of our less precise measurements were weighted with a factor of 5, while the earlier visual and photographic times (esp. the times of the mid-exposure of a photographic plate) were given a weight of 1 or 0 because of the large scatter in these data. We tested the stability of the results with respect to our - somewhat arbitrarily chosen - weighting scheme. It turned out that the results show some dependence on the weighting but this is mainly related to the less ideal distribution of available observations. For this reason, the results must be considered preliminary.

\subsection{V871 Aql}

The detached eclipsing binary V871 Aquilae (=SVS $1215=$ FL 2526; $\alpha_{2000}=18^{\mathrm{h}} 42^{\mathrm{m}} 32^{\mathrm{s}}, \quad \delta_{2000}=-3^{\circ} 10.0^{\prime}$, $V_{\max }=13.6$ mag; Sp. G2) is a neglected, rather faint eclipsing binary with eccentric orbit $(e=0.13)$ and an orbital period of about 3 days. It was discovered to be a variable star in the field of SA 110 by Kurochkin (1956), who derived the first light elements

Pri. Min. $=$ HJD $2432764.365+2.95300 \cdot$ E.

Later, Kurochkin (1979) improved the light elements with a shorter orbital period

Pri. Min. $=$ HJD $2438637.28+2$ d.952682 $\cdot$ E.

Since its discovery, no photometric, spectroscopic or period study of this star was published as far as we know. Ten new epochs were derived from the photographic measurements given in Kurochkin (1979). A total of 15 times of minimum light were used in our analysis, with 8 secondary eclipses among them.

The computed apsidal motion elements and their internal errors of the least squares fit (in brackets) are given in Table 2. In this table $P_{\mathrm{s}}$ denotes the sidereal period, $P_{\mathrm{a}}$ the anomalistic period, $e$ represents the eccentricity and $\dot{\omega}$ is the rate of periastron advance (in degrees per cycle or in degrees per year). The zero epoch is given by $T_{0}$ and the corresponding position of the periastron is represented by $\omega_{0}$. The $\mathrm{O}-\mathrm{C}$ residuals for all times of minimum with respect to the linear part of the apsidal motion equation are shown in Fig. 1. The non-linear predictions, corresponding to the fitted parameters, are plotted as continuous and dashed curves for primary and secondary eclipses, respectively.

\subsection{V345 Lac}

The detached eclipsing binary V345 Lacertae (=GSC 3986.2900 $=\mathrm{VV} 464=\mathrm{FL} 3354 ; \alpha_{2000}=22^{\mathrm{h}} 18^{\mathrm{m}} 43.4^{\mathrm{s}}, \delta_{2000}=+54^{\circ}$ $40^{\prime} 33.5^{\prime \prime}, V_{\max }=11.3 \mathrm{mag}$; Sp. B8) is the next relatively little known, early-type eccentric binary candidate for the detection of the apsidal motion. It has a high orbital eccentricity of $e \simeq 0.5$ and a longer period of about 7.5 days. It was discovered to be a variable star photographically by 


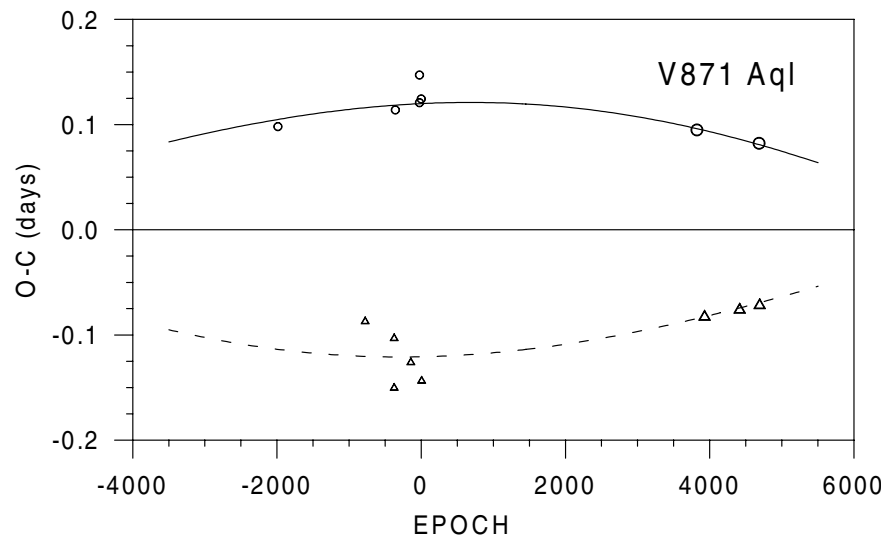

Fig. 1. The O-C diagram for the times of minimum of V871 Aql. The continuous and dashed curves represent predictions for the primary and secondary eclipses, respectively. The individual primary and secondary minima are denoted by circles and triangles, respectively. Larger symbols correspond to the photoelectric or CCD measurements which were given higher weights in the calculations.

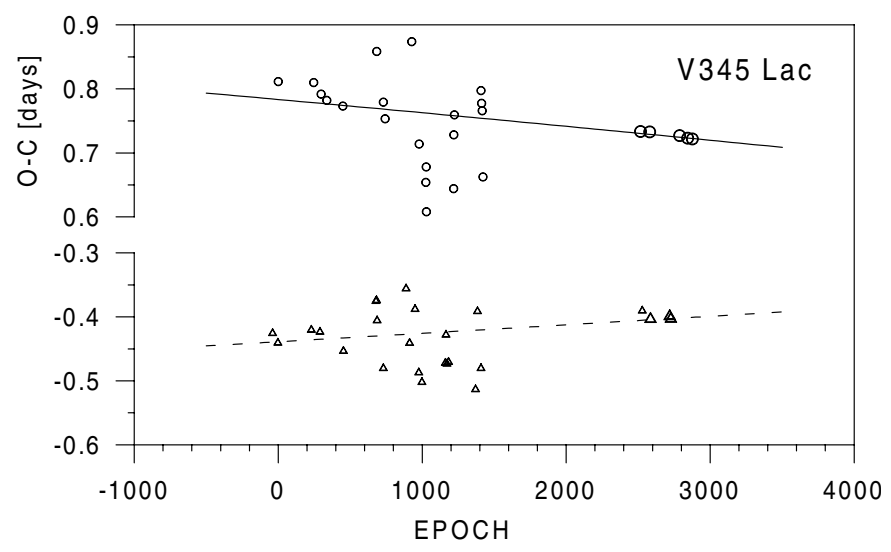

Fig. 2. O-C graph for V345 Lac. See legend for Fig. 1.

Miller \& Wachmann (1973), who obtained first different light elements for primary and secondary minimum

Pri. Min. $=$ HJD $2433569.493+7^{\mathrm{d}} .491746 \cdot \mathrm{E}$,

Sec. Min. = HJD $2433564.526+7.491761 \cdot E$,

and recommended this eclipsing variable for future apsidal motion study. The photographic light curve and new times of minimum light were later found by Busch (1978) from the plates of the Sonnenberg and Hartha Observatories. He derived the light elements with rather longer orbital period:

Pri. Min. $=$ HJD $2431344.407+7.491862 \cdot \mathrm{E}$, Sec. Min. $=$ HJD $2431346.954+7$ d.491862 $\cdot$ E.

He also announced the possibility of an apsidal motion in this system and found a relatively large value of the orbital eccentricity $e=0.50$ and $\omega=240 \mathrm{deg}$. Our new CCD times are given in Table 1. A total of 50 times of minimum light were used in our analysis, with 25 secondary eclipses among them. The resulting apsidal motion parameters are again given in Table 2. The $\mathrm{O}-\mathrm{C}$ residuals for all times of minimum with respect to the linear part of the apsidal motion equation are shown in Fig. 2 as explained above.

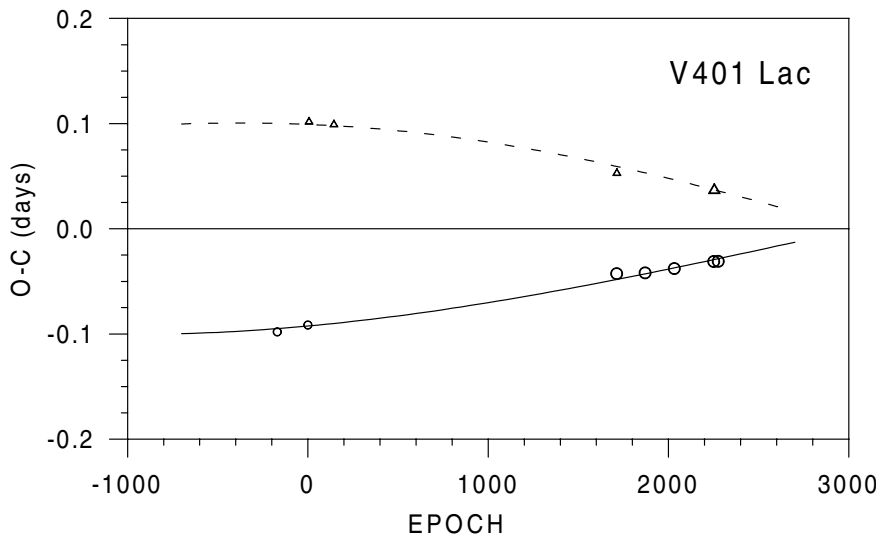

Fig. 3. The O-C diagram for V401 Lac. See legend for Fig. 1.

\subsection{V401 Lac}

The detached eclipsing binary V401 Lacertae (=HD 210308 $=$ HIP $109283=\mathrm{BD}+483621=\operatorname{ADS} 15661 \mathrm{~A} ; \alpha_{2000}=$ $\left.22^{\mathrm{h}} 8^{\mathrm{m}} 21.3^{\mathrm{s}}, \delta_{2000}=+49^{\circ} 13^{\prime} 15.6^{\prime \prime}, V_{\max }=7.9 \mathrm{mag} ; \mathrm{Sp} . \mathrm{A} 0\right)$ is a newly discovered and relatively bright early-type binary with an orbital eccentricity of $e=0.16$ and a short period of about 2 days. It was discovered to be a variable star by the ESA satellite Hipparcos - see Perryman et al. (1997) who also give the first light elements

Pri. Min. = HJD $2448501.7900+1$ d.95010 $\cdot$ E.

Bulut \& Demircan (2001) obtained a new $U B V$ light curve at Tübitak Observatory during 2000 and 2001. They also announced the possibility of a rapid apsidal motion in this system and predicted an apsidal-motion period of about 150 years. Our new CCD times of minima obtained at Ostrava in July and August 2001 are given in Table 1. Another primary minimum was obtained photoelectrically at Hvar Observatory in October 2003. The nearby comparison star HD $210119=\mathrm{BD}+48^{\circ} 3613\left(V=8^{\mathrm{m}} \cdot 35, B-V=0^{\mathrm{m}} \cdot 10\right.$, $\left.U-B=0^{\mathrm{m}} .08\right)$ was used during these $U B V$ observations which consisted of 10 -second integrations in each filter. They were carefully reduced to the standard $U B V$ system and corrected for differential extinction using the reduction program HEC 22 rel. 14 (Harmanec \& Horn 1998). The standard errors of these measurements were about $0.011,0.008$ and 0.006 mag in $U, B$ and $V$ filters, respectively. The time of the secondary minimum at HJD 2451843.418 was simply derived from the previous epoch using the phase of 0.55 given in the paper of Bulut \& Demircan (2001). Moreover, three new times were derived directly from the Hipparcos photometry data using the light-curve profile fitting method. Nevertheless, only 11 times of minimum light were collected in our preliminary analysis, with 4 secondary eclipses among them. The resulting apsidal motion parameters are again given in Table 2 . The $\mathrm{O}-\mathrm{C}$ residuals for all times of minimum with respect to the linear part of the apsidal motion equation are shown in Fig. 3 as explained above. 
Table 3. Eclipsing binaries with orbital eccentricities higher than 0.4 .

\begin{tabular}{cclcl}
\hline \hline System & $\begin{array}{c}\text { Spectral } \\
\text { type }\end{array}$ & $e$ & $\begin{array}{c}P \\
\text { [days] }]\end{array}$ & $\begin{array}{l}\text { Source } \\
\text { reference }\end{array}$ \\
\hline V883 Cen & B5 & 0.7 & 35.45 & Hensberge et al. (2004) \\
LV Her & F9 & 0.61 & 18.44 & Torres et al. (2001) \\
V1143 Cyg & F5+F5 & 0.540 & 7.641 & Giménez \& Margrave (1985) \\
DI Her & B4+B4 & 0.489 & 10.55 & Martynov \& Lavrov (1987) \\
V2283 Sgr & A0+A7 & 0.488 & 3.471 & Wolf (2000) \\
V541 Cyg & B9+B9 & 0.474 & 15.33 & Khaliullin (1985) \\
V345 Lac & B8 & 0.455 & 7.492 & this paper \\
MZ Lac & A0+A0 & 0.421 & 3.158 & Wolf et al. (1998) \\
V1647 Sgr & A1+A2 & 0.414 & 3.283 & Wolf (2000) \\
FT Ori & A0+A3 & 0.405 & 3.150 & Wolf \& Šarounová (1995) \\
\hline
\end{tabular}

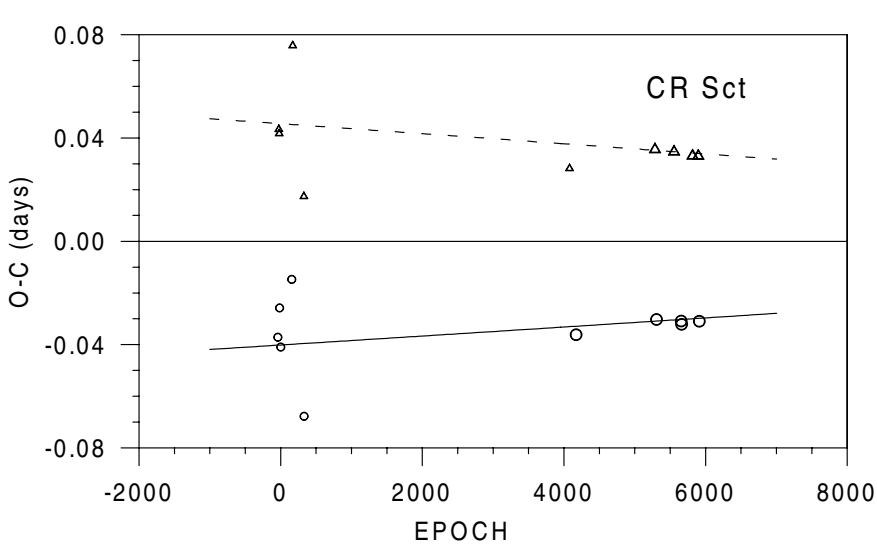

Fig. 4. O-C residuals for the times of minimum of CR Sct. See legend for Fig. 1.

\subsection{CR Sct}

The detached eclipsing binary CR Scutum (=GSC 5697.2825 $=$ FL 2561; $\alpha_{2000}=18^{\mathrm{h}} 48^{\mathrm{m}} 48^{\mathrm{s}}, \delta_{2000}=-9^{\circ} 30^{\prime} 11^{\prime \prime}, V_{\max }=$ 10.9 mag; Sp. A3) has also attracted little attention, being a rather faint early-type binary with a small orbital eccentricity $(e=0.09)$ and a period of about 4.2 days. Its variability was discovered photographically by Oosterhoff (1943), who obtained also the first light elements

Pri. Min. = HJD $2428069.626+4.192325 \cdot$ E.

As far as we know, this star has not been studied since then. All times of minimum light published by Oosterhoff (1943) and our new minima obtained at Tien-Shan and Ondřejov observatories were included, with the weighting scheme mentioned in Sect. 3. A total of 19 times of primary and secondary minima were used. Preliminary apsidal-motion elements are also given in Table 2, the corresponding O-C diagram is plotted in Fig. 4.

\section{Conclusions}

We discovered apsidal motion and derived new apsidal motion elements for four eccentric eclipsing binaries by means of an $\mathrm{O}-\mathrm{C}$ diagram analysis. The shortest period of apsidal motion among all discussed binaries was found for V401 Lac; the longest period was derived for V345 Lac.
Concerning the orbital parameteres, V401 Lac seems to be very similar to HS Her, which is another eclipsing binary with a relatively short orbital period of 1.64 days and a similar apsidal-motion period of about 78 years (Wolf et al. 2002). For V401 Lac, it is also highly desirable to obtain new, highdispersion and high-S/N spectroscopic observations and apply modern disentangling methods to them to obtain the radialvelocity curves of both binary components and, therefore, derive accurate masses for this important system.

Table 3 summarizes ten known eccentric eclipsing binaries with the eccentricity $e>0.4$. There is a small subgroup of EEBs of similar spectral types (about A0-A3), similar periods (between 3.0 and 3.5 days) and also values of the orbital eccetricity $(e \simeq 0.4)$ : MZ Lac, RU Mon, FT Ori, V1647 Sgr and V2283 Sgr.

Only a small part of the apsidal motion period for all these binaries is well covered by precise photoelectric and CCD observations. Therefore, new high-accuracy timings of these eclipsing systems are necessary to enlarge the timespan for a better analysis of the apsidal motion and to confirm the parameters given above. A spectroscopic orbit, allowing a precise mass determination, should also be obtained for a more thorough study and a more definitive determination of orbital and physical properties of all systems discussed in this paper.

Acknowledgements. Dr. T. Gráf, Johann Palisa Observatory, is acknowledged for his support of our CCD observations at Ostrava. This research has made use of the SIMBAD database, operated at CDS, Strasbourg, France, and of NASA's Astrophysics Data System Bibliographic Services. This investigation was supported by the Grant Agency of the Czech Republic, grant No. 205/04/2063, from the research plans J13/98: 113200004 of Ministry of Education, Youth and Sports and AV 0Z1 003909 and project K2043105 of the Academy of Sciences of the Czech Republic.

\section{References}

Ak, H., \& Filiz, N. 2003, IBVS, No. 5462

Bulut, I., \& Demircan, O. 2001, IBVS, No. 5179

Busch, H. 1978, Mitteilungen Bruno-H.-Bürgel-Sternwarte Hartha, No. 13,20

Diethelm, R. 1996, BBSAG Bull., 112, 5

Giménez, A. 1994, Exp. Astron., 5, 91

Giménez, A., \& Bastero, M. 1995, Ap\&SS, 226, 99 
Giménez, A., \& García-Pelayo, J. M. 1983, Ap\&SS, 92, 203

Giménez, A., \& Margrave, T. E. 1985, AJ, 90, 358

Harmanec, P., \& Horn, J. 1998, J. Astron. Data, 4, CD-ROM file 5, ed. C. Sterken (Brussel: Vrije Universiteit)

Hegedüs, T. 2000, private communication

Hegedüs, T., Borkovits, T., Bíró, I. B., et al. 2003, IBVS, No. 5372

Hensberge, H., Nitchelm, C., Freyhammer, L. M., et al. 2004, in Spectroscopically and Spatially Resolving the Components of Close Binary Stars, ASP Conf. Ser., ed. R. W. Hilditch, H. Hensberge, \& K. Pavlovski, in press

Khaliullin, Kh. F. 1985, ApJ, 299, 688

Khaliullin, Kh. F., Mironov, A. V., \& Moshkalyov, V. G. 1985, Ap\&SS, 111, 291

Kreiner, J. M., Kim, C.-H., \& Nha, I.-S. 2001, An Atlas of O-C diagrams of Eclipsing Binary Stars (Cracow Poland: Wydawnictwo Naukowe Akademii Pedagogicznej)

Kurochkin, N. E. 1956, Variable Stars, 11, 111
Kurochkin, N. E. 1979, Variable Stars Suppl., 3, 415

Kwee, K. K., \& van Woerden, H. 1956, Bull. Astron. Inst. Netherlands, 12, 327

Martynov, D. Y., \& Lavrov, M. I. 1987, Pisma Astron. Zh., 13, 218

Miller, W. J., \& Wachmann, A. A. 1973, Ric. Astr. Specola Vaticana, 8,18

Oosterhoff, P. Th. 1943, BAN, 9, No. 355, 383

Perryman, M. A. C, Høg, E., Kovalevsky, J., Lindegren, L., \& Turon, C. 1997, ESA SP-1200, The Hipparcos and Tycho Catalogues

Petrova, A. V., \& Orlov, V. V. 1999, AJ, 117, 587

Torres, G., Lacy, C. H. S., Guilbault, P. R., et al. 2001, IBVS, No. 5201

Wolf, M. 2000, A\&A, 356, 134

Wolf, M., \& Šarounová, L. 1995, A\&AS, 114, 143

Wolf, M., Diethelm, R., Kozyreva, V. S., \& Šarounová, L. 1998, A\&A, 334,840

Wolf, M., Harmanec, P., Diethelm, R., Hornoch, K., \& Eenens, P. 2002, A\&A, 383, 533 